\title{
Screening for pain in knee osteoarthritis: which question?
}

\author{
Sheila C O’Reilly, Ken R Muir, Michael Doherty
}

\begin{abstract}
Objective-To compare three questions on knee pain with respect to determined prevalence and associations with disability and structural change.

Methods-Postal survey to 4057 men and women aged 40-79 years. Knee pain was defined by three questions: (A) "Have you ever had pain in or around the knee on most days for at least a month? If so, have you experienced any pain during the last year?" (B) "Have you had pain within the last year in or around the knee that occurred on most days for at least a month?" (C) "Have you had knee pain on most days of the last month?" [American College of Rheumatology (ACR) criteria for knee osteoarthritis]. Disability was assessed with the SF-36 health status questionnaire. Radiographs (AP weight bearing and skyline) were obtained on a proportion ( $n=459)$ and graded for maximum osteophyte in any compartment.

Results-Prevalence of knee pain for questions A, B, and C were $28.3 \%, 25.3 \%$, and $19.3 \%$ respectively. Highest rates of disability were observed for question C (71.3\% compared with $\mathbf{6 0 . 9} \%$ for question A). There was no major difference between questions in terms of percentage with $\geq$ grade 1 osteophyte or $\geq$ grade 2 osteophyte. Sensitivity and specificity of each question for $\geq$ grade 1 osteophyte did vary, with question $A$ being most sensitive but least specific (58.7\% and $59.1 \%)$ and question $\mathrm{C}$ most specific $(\mathbf{7 2 . 7 \%})$ but least sensitive $(45.4 \%)$.
\end{abstract}

Conclusions-Estimates of knee pain and disability are influenced by even minor changes in question content. The ACR criteria question may be a better predictor of disability but is relatively insensitive for use in the community.

(Ann Rheum Dis 1996;55:931-933)

Osteoarthritis is a major contributor to morbidity in the community, and the knee is one of the commonest sites affected. Studies to date have predominantly defined knee osteoarthritis in terms of radiographic features. The discordance between symptoms and structural change is, however, well recognised. ${ }^{1}$ In addition there remains disagreement as to the degree of structural change that constitutes a definition of osteoarthritis. Placing emphasis on osteophytic change, as many favour, may exclude those with early disease. For these rea- sons, attention is now focusing on presence of symptoms. ${ }^{2}$ The presence of pain is an obligatory component of the American College of Rheumatism (ACR) criteria for knee osteoarthritis. ${ }^{3}$ Defining pain, however, may be just as problematic as defining structural change. Community studies assessing presence of pain have used less rigid definitions than the $\mathrm{ACR}^{45}$ - but even in these there has been little consistency in terms of question. What impact such variation may exert on results has not been assessed. The aim of this study was firstly to assess prevalence of knee pain in the community using different definitions of pain, and secondly to assess the effects of differing questions on levels of associated disability and on the relation of pain to structural change.

\section{Methods}

This study was approved by the local research ethics committee.

\section{SUBJECTS}

Lists of men and women aged 40-79 years were obtained from two general practices in Nottingham. A proportion was then selected by random sampling, stratified by age. Patients were excluded by their respective general practitioner if terminally ill, severely demented, or suffering from major psychiatric illness, with 53 excluded in total. The final population consisted of 4057 subjects (2096 women, 1961 men)

\section{POSTAL SURVEY}

This included questions concerned on basic demographic details in addition to the following knee pain questions:

(A) Have you ever had pain in or around the knee on most days for at least a month? If so, have you experienced any pain during the last year?

(B) Have you had pain within the last year in or around the knee that occurred on most days for at least a month?

(C) Have you had knee pain on most days of the last month?

Question A was used in a Bristol survey of knee pain. ${ }^{6}$ To be designated "knee pain" positive a positive response was required to both parts of the question. Question B is a modified version of $\mathrm{A}$, which ensures that the "month of pain" has occurred within the last year. Question C is the ACR criteria question for knee pain. The questions were asked in the same order in all questionnaires. The health status instrument SF-36 was included in the questionnaire, ${ }^{7}$ and 
Table 1 Prevalence of knee pain, disability and osteophyte with differing knee pain positivity and with no pain

\begin{tabular}{lllll}
\hline & $\begin{array}{l}\% \text { Knee pain } \\
(n)\end{array}$ & $\begin{array}{l}\% \text { Disability } \\
(n)\end{array}$ & $\begin{array}{l}\% \geq \text { grade 1 osteophyte } \\
(n)\end{array}$ & $\begin{array}{l}\% \geq \text { grade 2 osteophyte } \\
(n)\end{array}$ \\
\hline Question A & $28.3(905)$ & $62.3(556)$ & $74.0(179)$ & $40.9(98)$ \\
Question B & $25.3(811)$ & $54.9(515)$ & $76.0(158)$ & $42.3(88)$ \\
Question C & $19.3(618)$ & $71.7(434)$ & $76.7(138)$ & $43.3(78)$ \\
No pain & & $27.5(605)$ & $58.1(126)$ & $17.5(38)$ \\
\hline
\end{tabular}

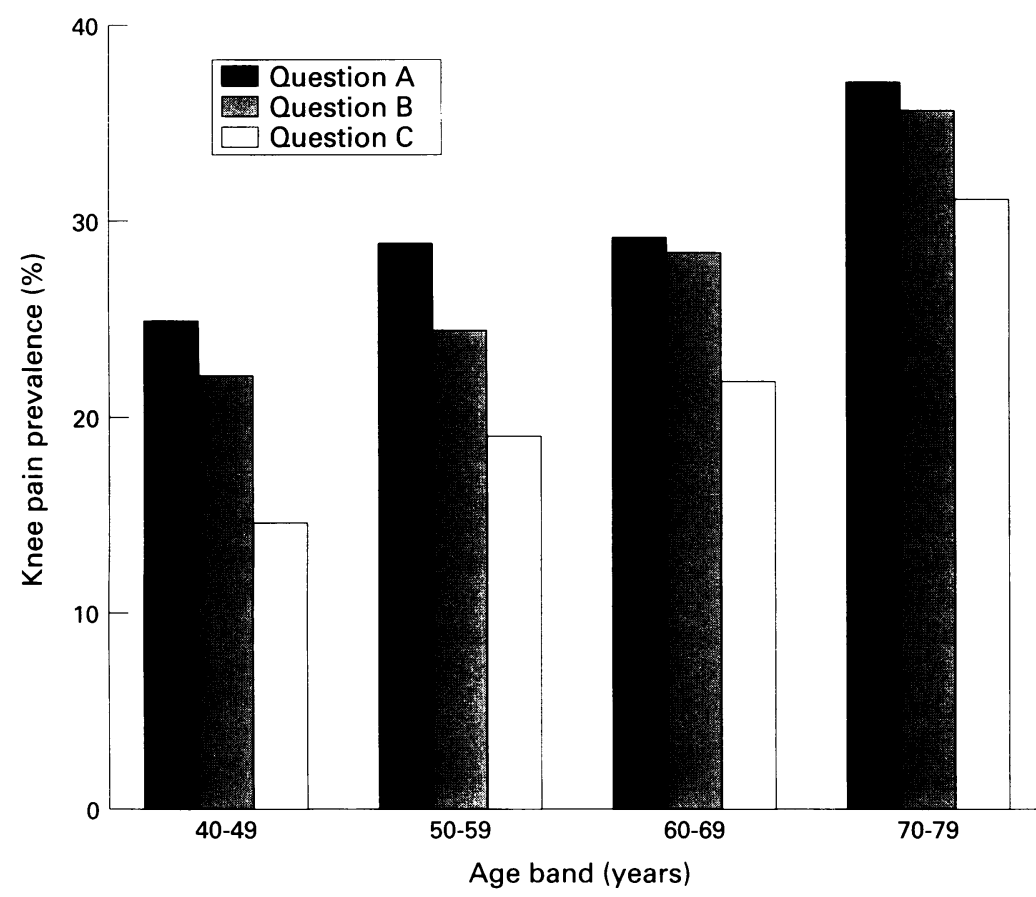

Figure 1 Knee pain prevalence with differing knee pain questions.

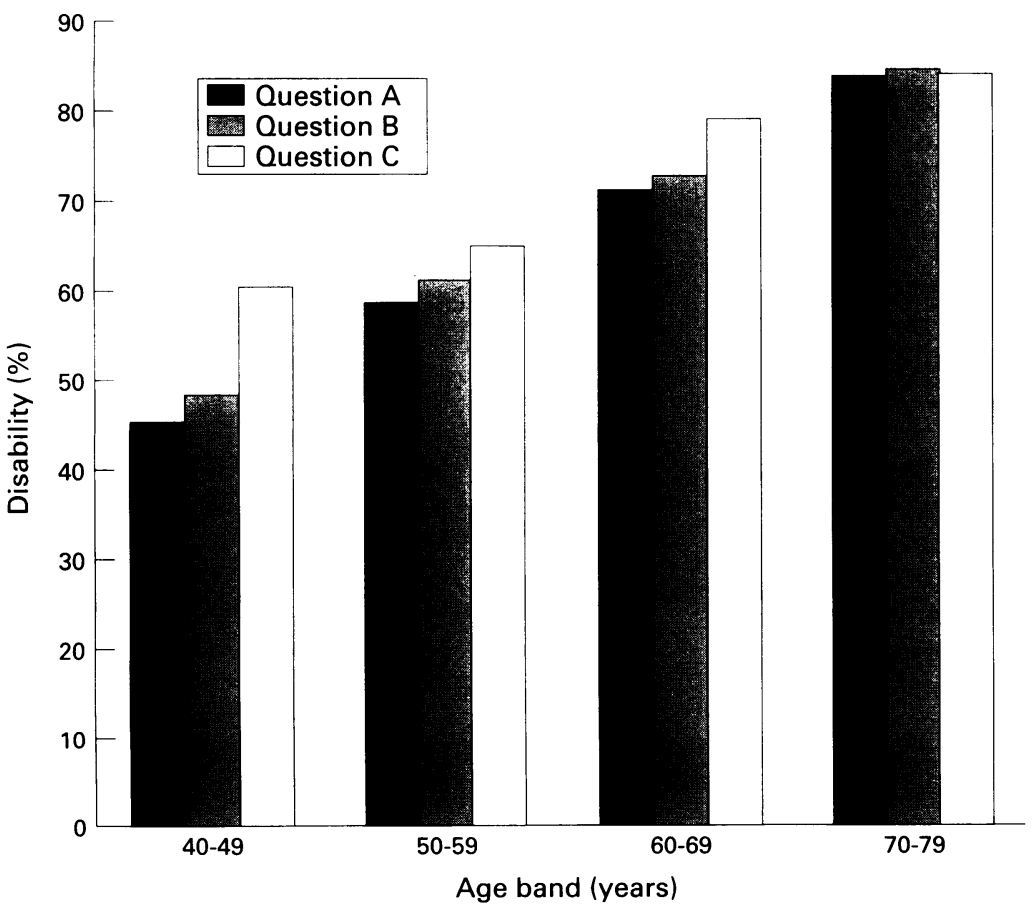

Figure 2 Prevalence of disability with differing knee pain questions.

the physical function dimension of this used to define disability. The dimension consists of 10 items, of which five must be answered to obtain a total score (0-100, with higher scores indicating better function). For this study disability was defined as a score within the lowest tertile of the total population.
RADIOGRAPHS

Standardised AP weight bearing (full extension) and skyline views (30 degrees of flexion) were obtained on a proportion of subjects with and without pain (as defined by question A). Radiographs were read blindly by one investigator using a standard atlas. ${ }^{8}$ Osteophytes were graded $0-3$ for all three compartments (medial tibiofemoral, lateral tibiofemoral, and patellofemoral). An overall osteophyte grade was assigned, being the maximum osteophyte grade for any compartment in either knee. Forty of the films, representing a spectrum of severity, were read twice. Reproducibility was assessed by calculating an unweighted $\kappa$ coefficient for osteophyte grade in each compartment. ${ }^{9}$

\section{Results}

PREVALENCE OF KNEE PAIN AND DISABILITY

A response rate of $81.9 \%$ was achieved following a reminder. Basic prevalence rates for each question differed as shown in table $1 ; 97.4 \%$ of those positive for question $\mathrm{C}$ were positive for question $\mathrm{A}$, and $94.8 \%$ were positive for question $\mathrm{B}$. Of those positive for question $\mathrm{B}, 94.8 \%$ were also positive for question A. From fig 1 it is clear that discrepancy in prevalence is greatest in the younger age groups. Despite the similarity between the first two questions, higher prevalence rates are consistently obtained with question B. Disability also varies with question, with highest levels associated with question $\mathrm{C}$. The difference is again less apparent in the older age groups (fig 2).

RELATION WITH OSTEOPHYTE

Radiographs were obtained on 459 subjects. Intraobserver reproducibility was good at all sites $(\kappa>0.8)$. From table 1 it can be seen that the percentage with $\geq$ grade 1 osteophyte varies little with each question (that is, the positive predictive value of each question is similar). Percentage with $\geq$ grade 2 osteophyte is lower but again similar for each question. Sensitivity and specificity are shown in table 2. If osteophyte grade $\geq 1$ is taken as the gold standard, question $\mathrm{A}$ had the highest sensitivity $(58.7 \%)$ but lowest specificity $(59.1 \%)$, with question $\mathrm{C}$ having the lowest sensitivity $(45.4 \%)$ and highest specificity $(72.7 \%)$. Defining structural disease by a higher grade of osteophyte ( $\geq$ grade 2 ), produces higher levels of sensitivity but lower levels of specificity. The trend within questions is similar to that for the lower grade of osteophyte.

\section{Discussion}

Knee pain prevalence is highest with question A, being similar to rates obtained from the Bristol community survey. ${ }^{6}$ Prevalence is clearly affected by question content. A lower prevalence for question $C$ is not unexpected since it requires current symptoms. It is interesting, however, that even a subtle change in wording, as with questions $A$ and $B$, can produce different estimates of pain. The effect of ordering on response is difficult to predict 
Table 2 Sensitivity and specificity of each question with respect to presence of osteophyte

\begin{tabular}{lllll}
\hline & \multicolumn{2}{l}{ Grade 1 osteophyte } & \multicolumn{2}{l}{$\geq$ Grade 2 osteophyte } \\
\cline { 2 - 5 } & Sensitivity (\%) & Specificity (\%) & Sensitivity (\%) & Specificity (\%) \\
\hline Question A & 58.7 & 59.1 & 72.2 & 55.6 \\
Question B & 51.8 & 67.5 & 64.2 & 62.7 \\
Question C & 45.4 & 72.7 & 57.4 & 68.3 \\
\hline
\end{tabular}

and may have influenced results. Any such effect might have caused omission of subsequent questions or an affirmative response to all three. Neither of these, however, occurred in excess of the other options, making a significant effect unlikely.

The results have implications in terms of interpreting previous population surveys. The knee pain questions from the NHANES and Framingham studies were not identical. NHANES took "ever" as the definition, ${ }^{4}$ with Framingham adding a further clause of this pain having occurred within the last two years. ${ }^{5}$ Framingham reported $29 \%$ with knee osteoarthritis having pain, while in the NHANES population, the corresponding figure was $41 \%$. While other differences such as radiological methods were important, the variation in question may have been a contributing factor. Doubling of knee pain rates have been previously reported when the ACR question is adjusted to any pain in the last month. ${ }^{10}$ Overall pain rates in this study were lower, but this was a smaller and younger population. It is interesting that in the current study differences in prevalence tailed off at the higher age groups. It may be that this represents more established knee disease with severe and constant symptoms. Alternatively the elderly age groups may be less careful at differentiating between subtle changes in question content.

The trend for disability is similar to that for prevalence. Perhaps not surprisingly, the ACR criteria question, which requires pain on most days of the last month, is associated with most impairment of function. While an arbitrary cutoff of physical function score was taken as defining disability, it is apparent that pain as defined by the other two questions is still associated with definite functional impairment. Using the ACR criteria alone may therefore omit a population with significant morbidity.

To assess the relation between pain and structural change, the presence of osteophyte was used. Clearly there is still much debate, with some advocating inclusion of joint space narrowing. With the growing trend away from the original Kellgren and Lawrence grades, ${ }^{11}$ it is important to standardise grading of individual features. The use of an atlas may aid such standardisation. The inclusion of the patellofemoral joint is important and there is increasing evidence in favour of its assessment by skyline views, in terms of both reproducibility and correlation with pain. ${ }^{1213}$ Which precise grade of osteophyte best defines osteoarthritis, however, remains speculative. The similarity of each question in this study in terms of their predictive value for osteophyte supports the poor relation between structural change and pain. The high prevalence of osteophyte in those without pain is additional confirmation. The questions do, however, vary in terms of their sensitivity and specificity for osteophyte. The ACR criteria, since less prevalent, are relatively insensitive though more specific. Although the selection process will have led to an increase in sensitivity of question $A$ with respect to osteophyte, it will not have altered sensitivity relative to the other two questions. In a population survey where the overall burden of pain or disability is the relevant variable to measure, questions $\mathbf{A}$ or $\mathbf{B}$ would seem more appropriate. The ACR criterion question should perhaps be reserved for use in hospital populations, or in therapeutic trials where current and potentially more severe symptoms are most relevant.

We are grateful to the Arthritis and Rheumatism Council for Research for funding this study, and are indebted to the general practitioners and patients of the Stenhouse Medical Centre, Arnold and the Calverton Surgery for their cooperation.

1 Lawrence JS, Bremner JM, Bier F. Osteo-arthrosis: prevalence in the population and relationship between symptoms lence in the population and relationship between sy

2 Hadler NM. Knee pain is the malady-not osteoarthritis. Ann Intern Med 1992;116:598-9.

3 Altman RD, Asch E, Bloch D, Bole G, Borenstein D, Brandt $\mathrm{K}$, et al. Development of criteria for the classification and reporting of osteoarthritis: classification of osteoarthritis of the knee. Arthritis Rheum 1986;29:1039-49.

4 Anderson J, Felson DT. Factors associated with osteoarthritis of the knee in the first national health and nutrition examination survey (HANES 1). Evidence for an associexion with overweight, and assical demands of work. Am 7 Epidemiol 1988,

5 Felson DT, Naimark A, Anderson JJ, Kazis L, Castelli W, Meenan RF. The prevalence of knee osteoarthritis in the Meenan RF. The prevalence of knee osteoarthritis in the
elderly: the Framingham osteoarthritis study. Arthritis elderly: the Framingh

6 McAlindon TE, Cooper C, Kirwan JR, Dieppe PA. Knee pain and disability in the community. $B r \mathcal{F}$ Rheumato 1992;31:189-92.

7 Brazier JE, Harper R, Jones NM, O'Cathain A, Thomas KJ, Usherwood T, et al. Validating the SF-36 health survey questionnaire: new outcome measure for primary care. BMF 1992;305:160-5.

8 Burnett S, Hart DJ, Cooper C, Spector TD. A radiographic atlas of osteoarthritis. London: Springer Verlag, 1994.

9 Cohen J. A coefficient of agreement for nominal scales. Educ Psychol Measure 1960;20:37-46.

10 Spector TD, Hart DJ, Leedham-Green M. The prevalence of knee and hand osteoarthritis in the general population using different clinical criteria: the Chingford study using different clinical criteria: the Chin

11 Kellgren JH, Lawrence JS. Atlas of standard radiographs. The epidemiology of chronic rheumatism. Oxford: Blackwell Scientific Publications, 1963.

12 Cicuttini FM, Baker J, Hart DJ, Spector TD. Choosing the best method for radiological assessment of patellofemoral osteoarthritis. Ann Rheum Dis 1996;55:134-6.

13 Jones AC, Ledingham J, McAlindon TE, Regan M, Hart DJ, MacMillan PJ, et al. Radiographic assessment of patel lofemoral osteoarthritis. Ann Rheum Dis 1993;52:655-8. 